\title{
Suicide gene therapy of rhabdomyosarcoma
}

\author{
PAWEŁ KONIECZNY*, MACIEJ SUŁKOWSKI*, BOGNA BADYRA, JACEK KIJOWSKI and MARCIN MAJKA
}

\author{
Department of Transplantation, Faculty of Clinical Immunology and Transplantation, \\ Jagiellonian University Medical College, 30-663 Krakow, Poland
}

Received July 13, 2016; Accepted September 26, 2016

DOI: 10.3892/ijo.2016.3824

\begin{abstract}
Rhabdomyosarcoma is the most common soft tissue sarcoma in childhood and young adulthood. Conventional treatment consisting of surgery, chemotherapy and radiotherapy can be insufficient, as long-term survival chances decrease dramatically when cancer recurrence occurs. Due to this fact, efficient treatment of this cancer is still a demanding issue, thus, novel and innovative therapies have to be considered as a part of combined treatment. In the present study, we present effective suicide gene therapy of rhabdomyosarcoma cell line Rh30 involving herpes simplex thymidine kinase (HSV-TK) and ganciclovir (GCV). Transduction of rhabdomyosarcoma cells using lentiviral vectors allowed efficient introduction of HSV-TK gene. In this study we proved high susceptibility of modified cells to ganciclovir resulting in eradication of cancer cells both in vitro and in vivo. Our data revealed strong gap junctional intercellular communication in examined cell line responsible for elimination of unmodified cells by bystander effect, even if HSV-TK-expressing cells comprise only $20 \%$ of cultured cells. Moreover, investigated approach is also efficient in vivo, where complete remission of tumors upon only 14 days of systemic administration of GCV can be observed. Obtained results suggest that HSV-TK suicide gene therapy is very promising concept in future clinical studies concerning rhabdomyosarcoma.
\end{abstract}

Correspondence to: Professor Marcin Majka, Department of Transplantation, Faculty of Clinical Immunology and Transplantation, Jagiellonian University Medical College, Wielicka 265, 30-663 Krakow, Poland

E-mail: mmajka@cm-uj.krakow.pl

${ }^{*}$ Contributed equally

Abbreviations: Cx, connexin; GCV, ganciclovir; GFP, green fluorescent protein; GJIC, gap junctional intercellular communication; HSV-TK, herpes simplex virus thymidine kinase; RMS, rhabdomyosarcoma

Key words: suicide gene therapy, rhabdomyosarcoma, HSV-TK, bystander effect, thymidyne kinase, ganciclovir

\section{Introduction}

Rhabdomyosarcoma (RMS) is the most common soft tissue sarcoma in childhood and young adulthood and accounts for $4-6 \%$ of all malignancies in this age group $(1,2)$. RMS protein expression profile is characteristic for striated muscle tissue and includes desmin, myosin, sarcomeric actin and myoglobin. Rhabdomyosarcoma cells commonly exhibit a characteristic sarcomeric banding pattern, similar to skeletal muscle, due to the arrangement of cytoskeletal elements in the cytoplasm. However, there are numerous examples of RMS occurrence in organs with no striated muscular elements such as gallbladder, prostate or urinary bladder. This fact leads to conclusion, that various lines of RMS may originate from primitive mesenchyme, which demonstrates tendency to myogenesis (3). Clinical features and molecular biology allow identifying two main subtypes of RMS (embryonal and alveolar) and less frequent anaplastic rhabdomyosarcoma. Embryonal rhabdomyosarcoma (ERMS) usually affects infants and children before 5 years of age and is the most common type of RMS. This subtype tends to occur in head and neck area, however, it can be found also in or around urinal bladder, vagina or prostate (4). Alveolar rhabdomyosarcoma (ARMS) affects all age group equally, however, due to the fact that ERMS is less common at older ages, there are proportionally more cases of this type in older children and teens. ARMS generally occurs in large muscles of the trunk, arms and legs. ARMS in comparison to ERMS grows faster and requires more intense treatment as it has worse prognosis (5).

Treatment for rhabdomyosarcoma consists of surgery, chemotherapy and radiotherapy. It has to be noted, that surgery might be difficult or even impossible due to localization of the tumor. Chemotherapy is based on actinomycin D, cyclophosphamide, doxorubicin, etoposide, ifosfamide or vincristine administration. In addition to chemotherapy, radiation therapy can be implemented to elevate treatment success rate (6). However, treatment of RMS is realized as conventional therapy, scientists have put efforts into finding new therapies such as immunotherapy using monoclonal antibodies (7). Despite the fact that RMS may be treated effectively, long-term survival chances decrease dramatically when cancer recurrence occurs. That is the reason for developing novel and more effective therapies against rhabdomyosarcoma. Gene therapy based on suicide gene selectively eradicating cancer cells could be the solution. The introduction of herpes simplex virus thymidine kinase 


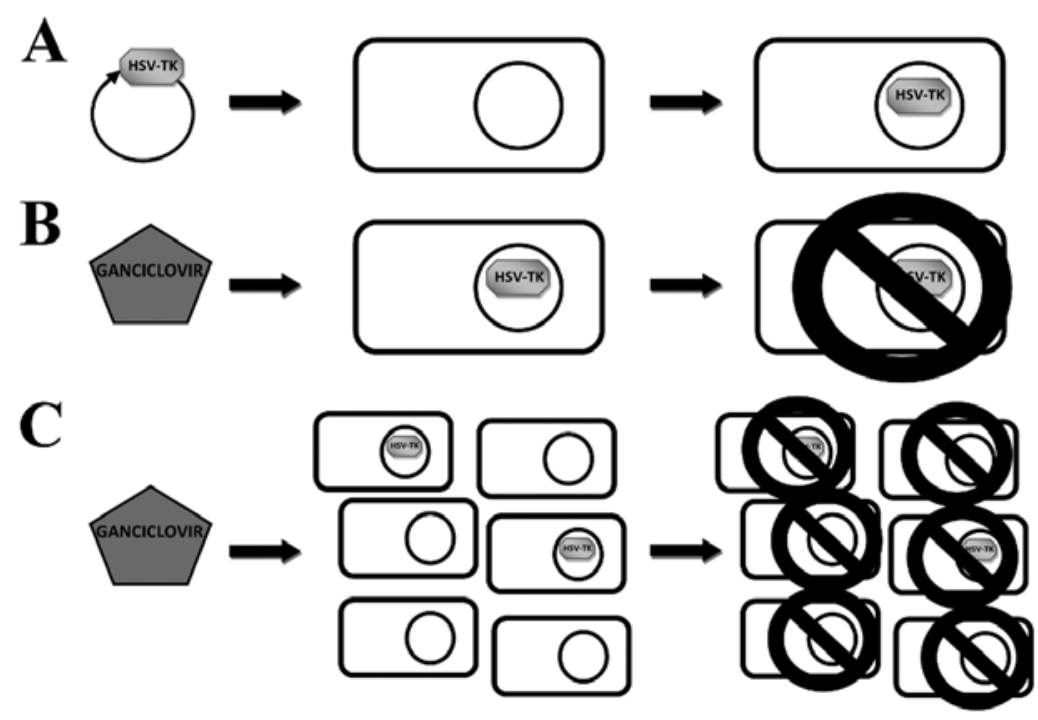

Figure 1. Schematic concept of suicide gene therapy. (A) Tumor cells are genetically modified either in vitro, in vivo or ex vivo by introduction of suicide gene (e.g. herpes simplex virus thymidine kinase, HSV-TK). (B) After administration of inactive prodrug ganciclovir, substrate for transgenic (HSV-TK) kinase, genetically modified cells metabolize the precursor to toxic metabolite (ganciclovir triphosphate). (C) Bystander effect occurs among mixed population of tumor cells. Ganciclovir toxic metabolite generated in cells expressing HSV-TK is transferred to neighbor (by standing) cells via gap junctions and leads to their death. Thus, all cells within the population (not only genetically modified) are eliminated in the course of suicide gene therapy.

(HSV-TK) allows to selectively eliminate cancer cells using antiviral medication, ganciclovir (GCV), a synthetic analogue of 2'-deoxy-guanosine.

The HSV-TK converts non-toxic ganciclovir into a toxic product and allows selective elimination of genetically modified, TK-expressing, cells in vitro and in vivo. Generally, thymidine kinase is the enzyme catalyzing the transfer of the $\gamma$-phosphate from ATP to thymidine to produce dTMP. Unlike the cellular thymidine kinase, herpes simplex virus thymidine kinase is able to process various substrates including pyrimidines and pyrimidine analogs (thymidine, deoxycytidine and AZT), as well as purine analogs (acyclovir, ganciclovir, buciclovir and penciclovir) (8). Ganciclovir (9-[(1,3-dihydroxy2-propoxy)methyl]guanine) is a potent inhibitor of viruses of the Herpes family, including cytomegalovirus (CMV). Ganciclovir is phosphorylated to ganciclovir monophosphate by HSV-TK and further by other cellular kinases. The final product of phosphorylation GCV is ganciclovir triphosphate, which is a competitive inhibitor of deoxyguanosine triphosphate (dGTP) that incorporates into DNA and, thus, inhibits DNA replication (8). Additionally, toxic GCV triphosphate can be effectively distributed to adjacent cells resulting in eradication of unmodified cells of the same type. This phenomenon is known as bystander effect (Fig. 1). Rendering neighbor cells similarly susceptible to ganciclovir is so efficient, that all tumor cells may be eliminated with as few as $10 \%$ of malignant cells expressing HSV-TK gene (9). The transport of activated prodrug to adjacent cells is realized through gap junctions (10). Gap junctions are clusters of intercellular channels built from connexins $(\mathrm{Cx})$ that allow direct diffusion of ions and small molecules (up to $2 \mathrm{kDa}$ ) between adjacent cells. Thus, they enable different biological functions including sustaining homeostasis, metabolites exchange and signaling (10-12).

Here, we present effective suicide gene therapy of RMS cell line Rh30 with application of ganciclovir. In the present study, tumor cells were efficiently eliminated both in vitro and in vivo. We also proved strong gap junctional intercellular communication (GJIC) in Rh30 cell line which contributes to lethal bystander effect in this cell line.

\section{Materials and methods}

Cell culture conditions. Rh30 rhabdomyosarcoma cell line was maintained as a monolayer culture in Dulbecco's modified Eagle's medium (DMEM) high glucose, containing glucose in concentration of $4.5 \mathrm{~g} / 1$ (Lonza, Basel, Switzerland) supplemented with $10 \% \mathrm{v} / \mathrm{v}$ fetal bovine serum (FBS; EURx, Gdańsk, Poland), $2 \mathrm{mM}$ L-glutamine and $100 \mathrm{U} / \mathrm{ml}$ penicillin and $100 \mu \mathrm{g} / \mathrm{ml}$ streptomycin (both from Thermo Fisher Scientific, Waltham, MA, USA) antibiotics solution. The cells were cultured at $37^{\circ} \mathrm{C}$ in a humidified atmosphere of $5 \% \mathrm{CO}_{2}$.

Generation and analysis of genetically modified cells. Lentiviral particles were generated accordingly to ViraPower protocol (Thermo Fisher Scientific) 9.5x106 HEK293T cells were seeded on T75 culture flask (DMEM high glucose, $10 \%$ FBS). Four hours after seeding, a transfection using calcium orthophosphate $\left(\mathrm{Ca}_{3}\left(\mathrm{PO}_{4}\right)_{2}\right)$ was performed. The transfection mixture was prepared as follows: 26-39 $\mu \mathrm{g}$ of plasmid DNA, $65 \mu \mathrm{l}$ of $2.5 \mathrm{M} \mathrm{CaCl}_{2}, 650 \mu \mathrm{l}$ of $2 \mathrm{X}$ BBS (BES buffered saline, $\mathrm{pH}$ 7.2) (both from Sigma-Aldrich, St. Louis, MO, USA) and $585 \mu \mathrm{l} \mathrm{H}_{2} \mathrm{O}$. The transfection mix was incubated for $20 \mathrm{~min}$ in room temperature, then transferred into fresh culture medium containing $25 \mu \mathrm{M}$ chloroquine (Sigma-Aldrich). Medium containing lentiviral particles was harvested 48 and $72 \mathrm{~h}$ after transfection. Harvested medium was centrifuged (1750 g, $10 \mathrm{~min}, 4^{\circ} \mathrm{C}$ ), filtered through $0.45 \mu \mathrm{m}$ syringe filter and frozen at $-80^{\circ} \mathrm{C}$. Two different transfection mixtures were prepared. eGFP@pLenti6 expression plasmid and pLP1,pLP2,pVSVG (Thermo Fisher Scientific) packaging plasmids were used to generate virus introducing green fluorescent protein (GFP). The second mix contained pLOX-gfp-iresTK expression 
plasmid with pCMV-dR8.2dvpr and pVSVG packaging plasmids. pLOX-gfp-iresTK plasmid was a gift from Didier Trono (Addgene plasmid \#12243). The same copy number, $1.5 \times 10^{12}$, of expression plasmids were used in both mixes.

To determine the titer of lentiviral stocks HT1080 cells were infected. Four hours prior to transduction $5 \times 10^{4}$ cells were seeded on a 24-well plate. Various volumes of harvested media (0.1-100 $\mu \mathrm{l})$ were added to seeded HT1080 cells. Fortyeight hours after the transduction the percentages of modified (GFP-expressing) cells were assessed by FACSCanto flow cytometer (Becton-Dickinson, Franklin Lakes, NJ, USA) to calculate transduction units (TU) in $1 \mathrm{ml}$ of each harvested medium.

To obtain Rh30 modified cell lines, cells were infected using generated lentiviral particles introducing GFP and TK gene (Rh30TK cell line) or GFP only in the Rh30GFP control cell line. Transduction was performed with MOI 5 (multiplicity of infection) and $6 \mu \mathrm{g} / \mathrm{ml}$ polybrene (Sigma-Aldrich). Rh30GFP and Rh30TK cell lines were purified using FACSAria II cell sorter (Becton-Dickinson). The percentage of green fluorescent cells in modified cell lines was measured by FACSCanto flow cytometer.

Suicide gene therapy in vitro. Rh30 $\left(2.5 \times 10^{4}\right)$ (WT, GFP and TK) cells were seeded in the wells of a 24-well plate. Four hours after seeding medium was changed for medium containing $0.1 \mu \mathrm{g} / \mathrm{ml}$ ganciclovir or control fresh medium. Each well was prepared in triplicate. On each of the following 5 days cells in each group (WT, GFP and TK \pm GCV) were collected and counted in Bürker's chamber. On the final day of the experiment cells were also photographed. In preliminary experiments 3 different GCV concentrations were tested, 10, 1 and $0.1 \mu \mathrm{g} / \mathrm{ml}$ (data not shown). Two different concentrations, 0.1 and $1 \mu \mathrm{g} / \mathrm{ml}$, were selected for further experiments (as being able to eliminate most of the HSV-TK expressing cells within several days while not being toxic to wild-type cells).

Gap-junctional intercellular communication (GJIC) and bystander effect. GJIC was investigated by intercellular transfer of calcein (13). Briefly, donor cells were stained with calcein $\mathrm{AM}$, green dye which may diffuse through gap junctions, and DiI (1,1-dioctadecyl-3,3,3,3-tetramethylindocarbocyanine perchlorate), red dye staining cell membranes, not transferred by gap junctions (both dyes from Thermo Fisher Scientific). Acceptor cells were seeded 24 to $48 \mathrm{~h}$ before the experiment and cultured until reaching $80-90 \%$ confluency. Donor cells were harvested and incubated in suspension in $1 \mathrm{mg} / \mathrm{ml}$ glucose solution containing $5 \mu \mathrm{M}$ calcein AM and $9 \mu \mathrm{M}$ DiI for $30 \mathrm{~min}$ in $37^{\circ} \mathrm{C}$ in darkness. During incubation cells were mixed several times. After triple wash with PBS (Lonza) stained donor cells were seeded onto monolayer of acceptor cells. After 90 min of incubation dye transfer was examined with a fluorescent microscope.

Bystander effect defined as transfer of toxic ganciclovir metabolite through gap junctions of neighboring cells was analyzed by co-culture of Rh30TK and Rh30WT cells. Mixtures of these cells were prepared in such manner that between $0 \%$ (Rh30WT only) and 80\% (Rh30TK only) of HSV-TK-expressing cells were present. Mixtures were seeded in duplicate. Twenty-four hours after seeding half of the wells received $1 \mu \mathrm{g} / \mathrm{ml}$ ganciclovir. The other half of the wells served as control. Cells were cultured for 8 days before analysis.

Xenografts and suicide gene therapy in vivo. All animal experiments were conducted according to the local ethics committee guidelines. Rh30 (WT, GFP and TK) cells were subcutaneously injected into adult female NOD/SCID mice. Cells $\left(5 \times 10^{6}\right)$ in $200 \mu$ of PBS were injected per mouse. Growth of tumors, and the health of the mice were monitored every day. When tumors become measurable mice in each group were randomly divided into two regimens: receiving $1 \mathrm{mg}$ of ganciclovir in $0.5 \mathrm{ml} \mathrm{PBS} \mathrm{(50} \mathrm{mg/kg} \mathrm{of} \mathrm{body} \mathrm{weight)} \mathrm{and}$ receiving only $0.5 \mathrm{ml}$ PBS. Both groups were injected intraperitoneally, daily for 14 days. After this period the mice were sacrificed, the tumors and the internal organs (liver, kidney, spleen, lungs, brains and heart) were isolated and fixed in $40 \%$ paraformaldehyde (Sigma-Aldrich). Tumors were measured and weighed. Fixed tissues were imbedded in paraffin, cut, stained with hematoxylin and eosin (H\&E) and analyzed histopathologically. Volume (v) of tumors was calculated with the equation $\mathrm{v}=1 / 2 \mathrm{ab}^{2}$ where $\mathrm{a}$ and $\mathrm{b}$ are tumor sizes.

Statistical analysis. Statistical analysis of acquired data was performed with STATISTICA 10.0 software (StatSoft, Inc., Tulsa, OK, USA). Student's t-test or one-way ANOVA with post-hoc Tukey's test were applied to verify statistically relevant differences between the experimental groups. Data are presented as mean \pm standard deviation. $\mathrm{P}<0.05$ indicates statistically significant difference.

\section{Results}

Genetic modification of rhabdomyosarcoma Rh30 cell line. In order to acquire rhabdomyosarcoma cells expressing herpes simplex virus thymidine kinase (HSV-TK) we performed stable lentiviral transduction of Rh30 cell line. Lentiviral vectors were produced in HEK293T cells based on Gateway ${ }^{\circledR}$ system. Cells were infected with multiplicity of infection MOI 5. Two cell lines were generated: Rh30GFP, expressing green fluorescent protein (GFP), and Rh30TK, expressing GFP and HSV-TK. The latter was established thanks to application of bicistronic lentiviral plasmid acquired from Addgene repository. Two cistrons are separated by internal ribosome entry site (IRES) which allows simultaneous expression of two transgenes. Lentiviral plasmid coding GFP was cloned in our laboratory previously. In both vectors expression of transgenes is regulated by strong promoter of cytomegalovirus (CMV). As the used bicistronic vector does not convey eukaryotic antibiotic resistance gene we performed fluorescence-activated cell sorting for purification of transduced cells. Hence we sorted GFP-positive cells and examined their purity by flow cytometry. After two rounds of cell sorting we acquired two cell lines, named Rh30GFP (Fig. 2A) and Rh30TK (Fig. 2B), with satisfactory purity, 93 and $80 \%$ of positive cells, respectively. Genetically modified cells had strong GFP expression (visible in microphotographs and histogram in Fig. 2) and typical morphology for rhabdomyosarcoma, although Rh30TK cell line was characterized by gently altered morphology (visible in Fig. 2B). Cells appeared to have slightly larger size with fewer cells elongated compared to wild-type cells (Rh30WT) 


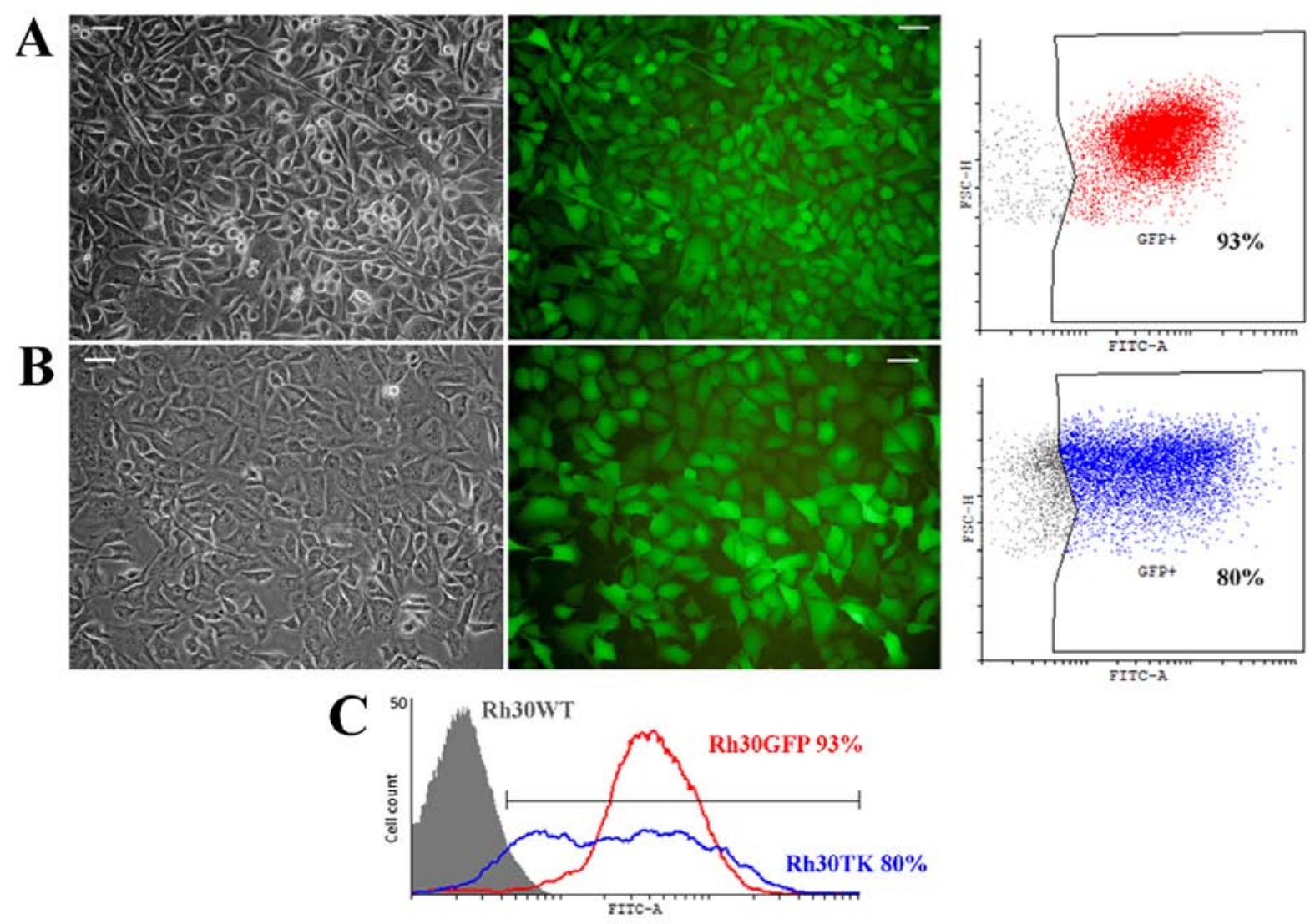

Figure 2. Generation of genetically modified rhabdomyosarcoma cell lines. Developed lines Rh30GFP (A, expressing GFP under CMV promoter) and Rh30TK (B expressing GFP and TK under CMV promoter) efficiently express transgenes and present high purity, 93 and $80 \%$, respectively, which can be verified on flow cytometry histogram (C). R30WT, wild-type Rh30 cells. Microphotographs magnitude in A and B, x200, white bars represent $50 \mu \mathrm{m}$.

and Rh30GFP cell line. We assumed that the phenomenon was caused by presence of exogenous kinase (TK) which might non-specifically phosphorylate multiple targets causing e.g. change in morphology.

Genetically modified rhabdomyosarcoma cells are highly vulnerable to ganciclovir. Our main goal was to evaluate the efficiency of suicide gene therapy in rhabdomyosarcoma. We used generated cell lines to assess vulnerability of HSV-TKexpressing rhabdomyosarcoma cells to ganciclovir. In preliminary experiments we investigated influence of different concentrations of ganciclovir (GCV) to rhabdomyosarcoma cells and selected value which was totally safe for unmodified cells, but was able to eradicate all HSV-TK-expressing cells within several days (data not shown). Upon 6 days of treatment of Rh30TK cells with $0.1 \mu \mathrm{g} / \mathrm{ml}$ ganciclovir virtually all HSV-TK-positive cells were eliminated (Fig. 3A) showing high level of toxicity of GCV for this cell line. Rh30TK cells grew normally in the absence of ganciclovir. Interestingly, this concentration was totally non-toxic to other cell lines, Rh30GFP and wild-type Rh30WT cells. Moreover, Rh30GFP cells expressed almost identical proliferation rate as Rh30WT cells despite the presence or absence of ganciclovir (Fig. 3B) proving that applied concentration of ganciclovir is neutral for cells lacking HSV-TK expression. Analysis of generated cell proliferation rate revealed that Rh30TK cells (without GCV in medium) were characterized by significantly slower growth rate compared to control cell lines (Rh30WT and Rh30GFP). This observation was associated with alterations in morphology and size of the HSV-TK expressing cells and might be explained by non-specific phosphorylation of signaling proteins involved in cell cycle, cell proliferation and cell growth performed by HSV-TK (see Discussion). Nevertheless, the data clearly proves that HSV-TK-expressing cells are efficiently and selectively eliminated by ganciclovir in low concentrations, which were non-toxic to wild-type cells. Thus, introduction of TK to rhabdomyosarcoma cells sensitizes rhabdomyosarcoma cells to GCV proving efficiency of applied suicide gene therapy in vitro in rhabdomyosarcoma.

Gap-junctional intercellular communication (GJIC) and toxic bystander effect in rhabdomyosarcoma. In our in vitro model virtually all HSV-TK-expressing cells were eliminated by ganciclovir treatment. It has to be noted that the population of genetically modified cells was very pure (reaching $80 \%$ of HSV-TK-positive Rh30 cells) meaning that vast majority of cells in culture expressed thymidine kinase. This might not be the case in experimental study concerning clinical use of suicide therapy. In situ (genetic modification of tumor cells directly in tumor) or ex vivo (isolated tumor cells or tumor infiltrating cells are modified in vitro and subsequently injected back to organism) approaches imply limited efficiency of cell transduction and, thus, considerably low percent of HSV-TKexpressing cells present in the tumor. To assess whether our approach could be effective in such situation we decided to examine the minimal proportion of Rh30TK cells in co-culture 


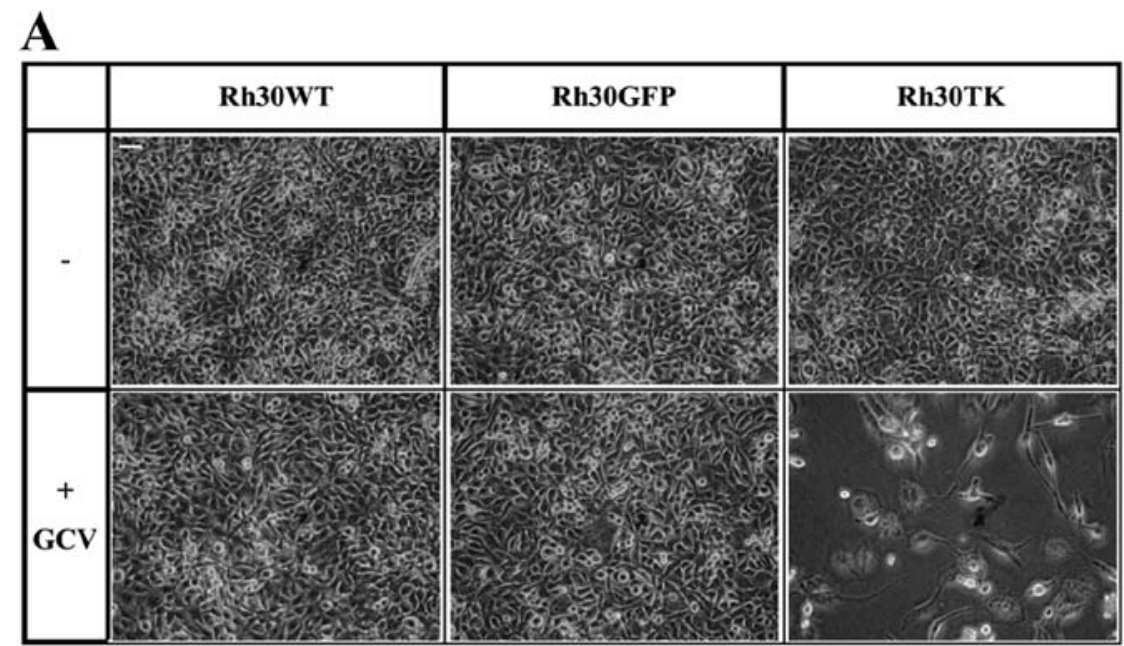

B

\section{Cell proliferation rate}

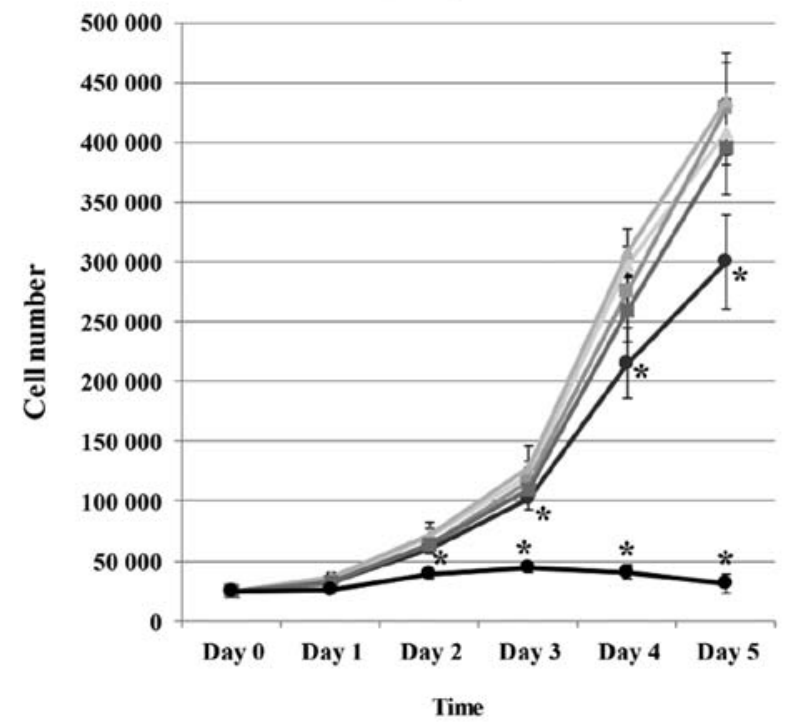

- Rh30WT

- Rh30GFP

Rh30TK

- Rh30WT

$+\mathrm{GCV}$

$\rightarrow-$ Rh30GFP

$+\mathrm{GCV}$

$\rightarrow$ Rh30TK

$+\mathrm{GCV}$

Figure 3. Ganciclovir efficiently eliminates HSV-TK expressing rhabdomyosarcoma cells. (A) After 6 days of treatment with $0.1 \mu \mathrm{g} / \mathrm{ml}$ ganciclovir (GCV) virtually all TK-expressing cells were eliminated while control cells (Rh30WT and Rh30GFP) remained intact. (B) Proliferation rate of generated cell lines in presence $(+\mathrm{GCV})$ or absence of ganciclovir. Data are presented as mean \pm standard deviation. ${ }^{*} \mathrm{P}<0.05$ indicates statistically significant difference vs. appropriate control tested by ANOVA, $\mathrm{n}=8$. Microphotographs magnitude in A, x200, white bar represents $50 \mu \mathrm{m}$.

necessary to effectively eradicate the whole co-culture of cells. HSV-TK-lacking cells are eliminated in co-culture thanks to ganciclovir toxic metabolite transfer through gap junctions of adjacent cells (Fig. 1C).

Firstly, we examined degree of gap junctional intercellular communication (GJIC) in Rh30 cell line. In classic experiment using double staining of donor cells with calcein (green dye transferred by gap junctions) and DiI (red dye staining cell membranes, not dissociating through gap junctions) we showed strong GJIC in rhabdomyosarcoma Rh30 cell line (Fig. 4). Unstained acceptor cells efficiently acquired calcein from stained donor cells showing effective transfer of low mass molecules between rhabdomyosarcoma cells through gap junctions.

Knowing that GJIC is efficient in Rh30 cell line we prepared mixtures of Rh30TK cells with Rh30WT containing between 10 and $80 \%$ of HSV-TK-expressing cells. After seeding and 8 days of treatment with ganciclovir we observed almost complete elimination of all cells in co-cultures containing $20 \%$ or more HSV-TK-positive cells (Fig. 5). We observed no toxicity in co-cultures without ganciclovir treatment. In mixture containing $10 \%$ of cells we observed only single cells left proving that small number of HSV-TKexpressing cells is sufficient to eliminate whole population of cells in mixture and thus, strong toxic bystander effect in rhabdomyosarcoma.

Suicide gene therapy in vivo is an efficient model. Efficient ganciclovir-induced elimination of HSV-TK-expressing cells in vitro and strong toxic bystander effect in Rh30 cell line are promising for clinical application of suicide gene therapy. To further confirm this notion we performed pre-clinical in vivo study in a xenograft model. After formation of measurable tumors in NOD/SCID mice we started daily intraperitoneal administration of ganciclovir in experimental groups and saline in control groups. Administration of $50 \mathrm{mg} / \mathrm{kg}$ ganciclovir 

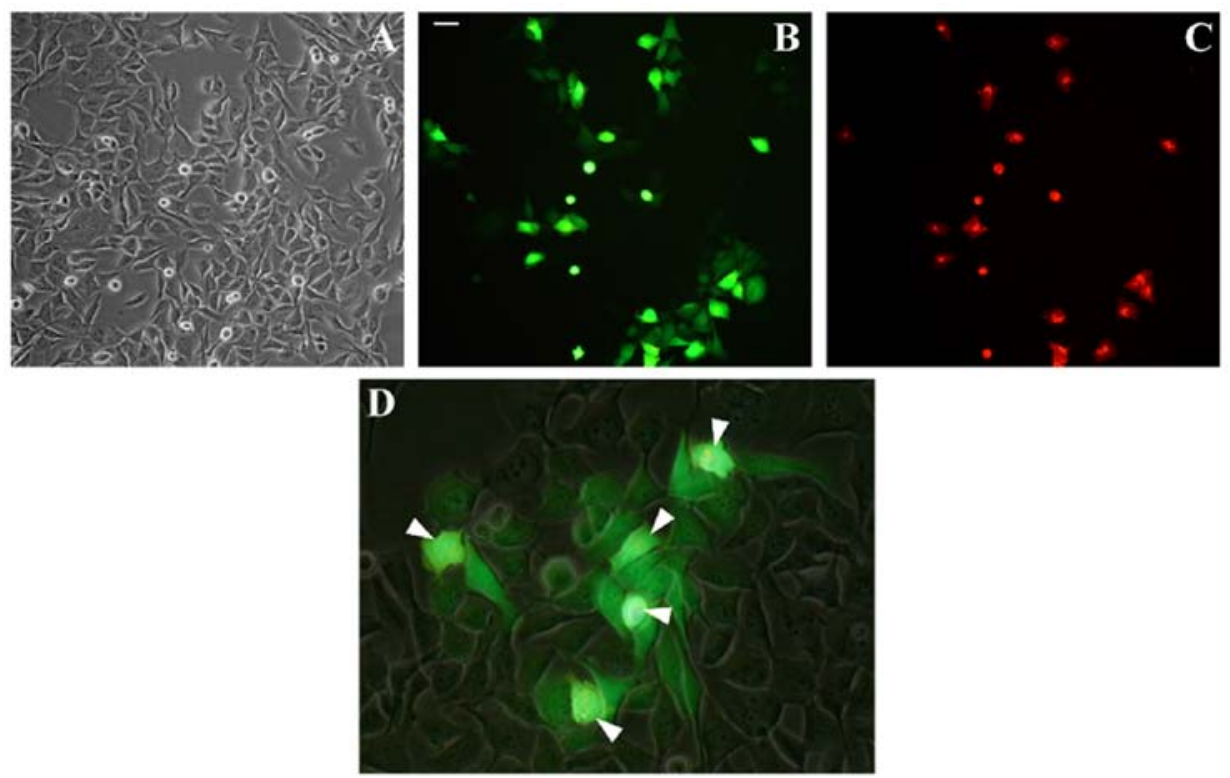

Figure 4. Gap-junctional intercellular communication (GJIC) in rhabdomyosarcoma Rh30 cell line. GJIC was examined by intercellular transfer of calcein between neighboring donor (stained red with DiI in C and pointed with arrowheads in D) and acceptor cells (light green in B and D). (A) Corresponding area in phase contrast. (B) Donor cells stained green transfer calcein to neighboring cells. (C) Donor cells stained red with DiI which did not cross gap junctions. (D) Merged photographs with donor cells marked with arrowheads. Microphotographs A, B, C and D magnitude, x200, white bar represents $50 \mu \mathrm{m}$.

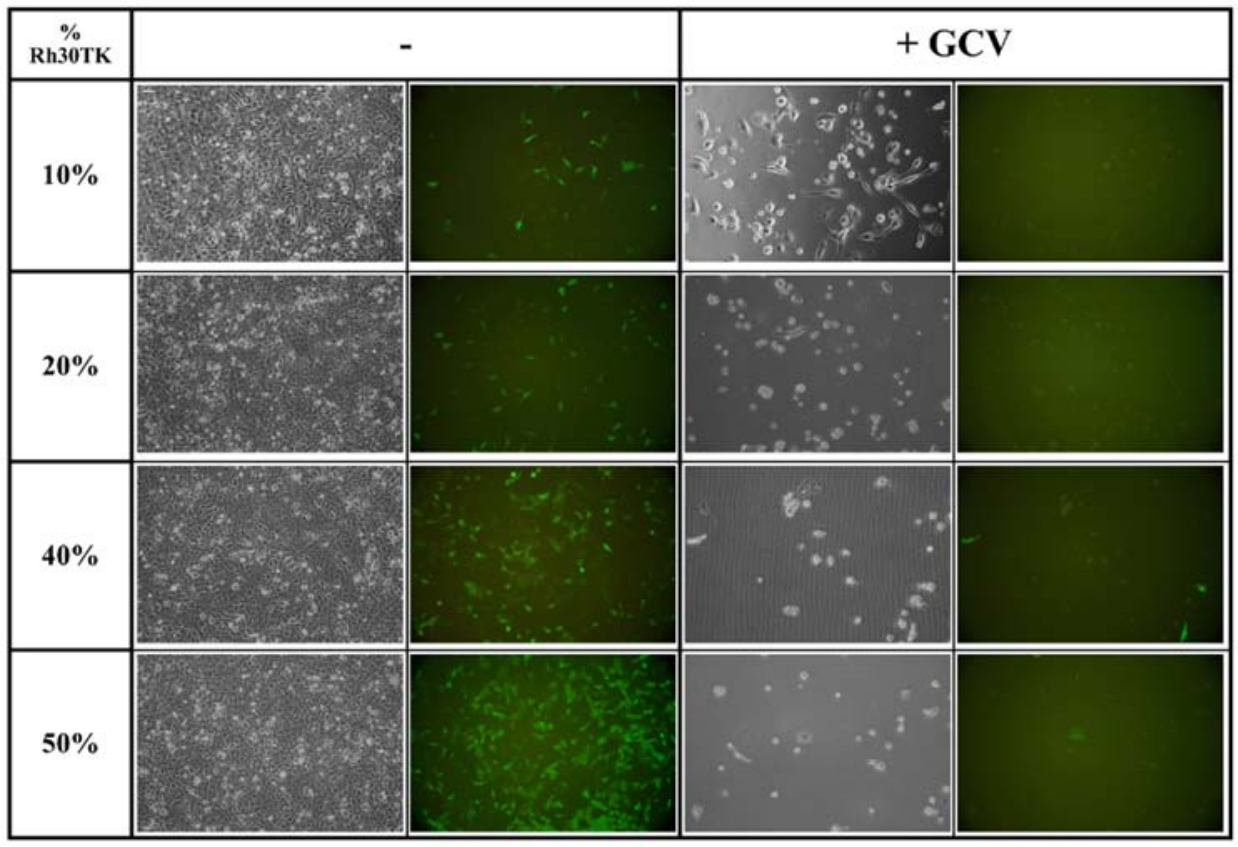

Figure 5. Toxic bystander effect in rhabdomyosarcoma Rh30 cell line. HSV-TK-expressing cells (Rh30TK) were mixed with wild-type cells to acquire appropriate concentrations (marked as percentage) of Rh30TK cells in populations. Co-cultures were maintained in absence (-) or presence of $1 \mu \mathrm{g} / \mathrm{ml} \mathrm{ganciclovir}$ (+GCV) for 8 days. As little as $20 \%$ of HSV-TK-expressing cells in population is enough to completely eradicate all cells in culture proving strong bystander effect in rhabdomyosarcoma Rh30 cell line. All microphotographs magnitude, x200, white bar represents $50 \mu \mathrm{m}$.

caused almost complete eradication of tumor formed by Rh30TK cells within 14 days of treatment (Fig. 6). In all other groups tumors developed at a rate comparable to wild-type cells. After 14 days mice were sacrificed and the tumors as well as the organs were isolated for histological analysis. It is noteworthy that in the course of daily systemic ganciclovir administration no multi-organ toxicity was observed (data not shown) suggesting that applied dosage of ganciclovir is neutral to healthy (not expressing HSV-TK) tissues and organs within applied time course. Histological analysis of isolated 'tumors' from $\mathrm{Rh} 30 \mathrm{TK}+$ ganciclovir group revealed that isolated tissues were actually lymph nodes with only single tumor cells (Fig. 6F). This observation proves efficient elimination of HSV-TK-expressing tumor cells in the in vivo model and encourages further pre-clinical research, which can bring suicide gene therapy concept to clinical applications. 

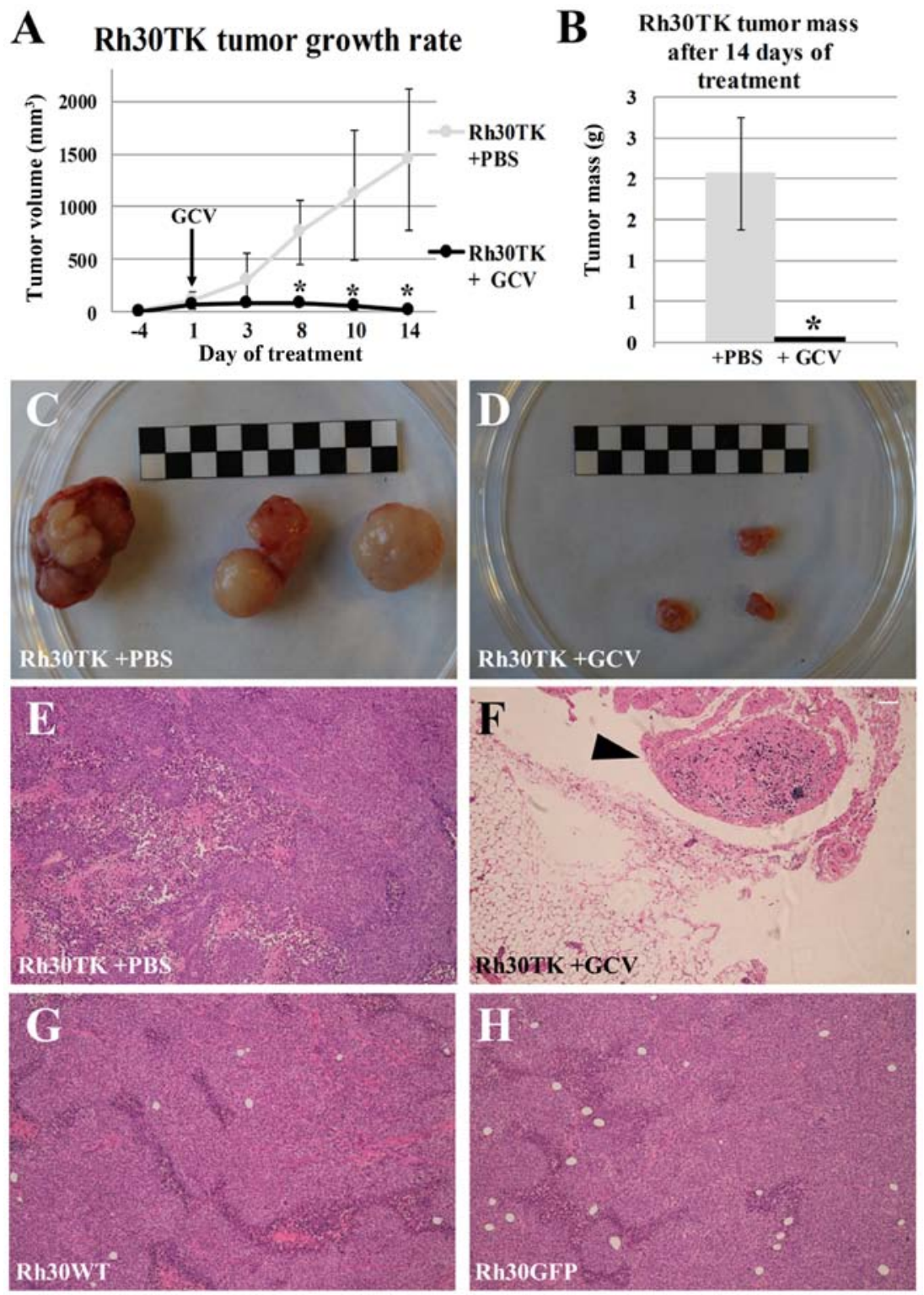

Figure 6. Suicide gene therapy of rhabdomyosarcoma in vivo. Tumors formed by Rh30TK cells in xenograft model were eradicated by ganciclovir treatment. After formation of tumors mice were daily injected with saline (PBS) or $50 \mathrm{mg} / \mathrm{kg}$ ganciclovir (GCV) intraperitoneally for 14 days. (A and B) Growth of Rh30TK tumor is abrogated after administration of ganciclovir (start of treatment is pointed by arrow). Charts respectively present increase of volume and final mass of tumors illustrated in C and D. Histopathological analysis of hematoxylin and eosin stained tumors (E, F, G and H) showed no differences in morphology between tumors formed by wild-type rhabdomyosarcoma cells (Rh30WT, G) or genetically modified cells, both Rh30GFP (H) and Rh30TK (E). Remnants of tumors isolated from Rh30TK+GCV group appeared to be lymph nodes (pointed with arrowhead in F) containing single tumor cells. Data in $\mathrm{A}$ and $\mathrm{B}$ are presented as mean \pm standard deviation. ${ }^{\mathrm{P}}<0.05$ indicates statistically significant difference vs. appropriate control tested by ANOVA (A) or Student's t-test (B), $\mathrm{n}=3$. White and black squares in C and D represent $5 \mathrm{~mm}$. Microphotographs $\mathrm{E}, \mathrm{F}, \mathrm{G}$ and $\mathrm{H}$ magnitude, $\mathrm{x} 100$, white bar represents $100 \mu \mathrm{m}$.

\section{Discussion}

Suicide gene therapy using herpes simplex virus thymidine kinase (HSV-TK) expression combined with ganciclovir (GCV) exposure is a well-known model in vitro. Our results show high efficacy of Rh30 rhabdomyosarcoma cell line elimination by suicide gene therapy involving HSV-TK and GCV. Virtually all HSV-TK expressing cells are eliminated within only 8 days of low dose $(0.1 \mu \mathrm{g} / \mathrm{ml})$ ganciclovir treatment. Genetic modification of cells with HSV-TK gene is claimed to have no impact on cell viability and proliferation capacities (14). Surprisingly, we observed significantly slower growth of Rh30TK cells without ganciclovir addition (Fig. 3). Additionally, no growth inhibition has been observed for control Rh30GFP cell line that proliferated as wild-type Rh30 suggesting that genetic modification is not responsible for decrease in proliferation rate of Rh30TK cell line. That decrease may be explained by HSV-TK ability to process wide range of substrates contrary to cellular thymidine kinases. Although, no ganciclovir was present in culture medium, the variety of other chemical compounds both in medium and within the cell could be potentially processed by HSV thymidine kinase, thus, an unspecified product of HSV thymidine kinase activity could interfere with cell cycle signaling pathway. Among many substrates of HSV-TK, some of them may be proteins involved in cellular signaling pathways (cyclins). Such deregulation could lead to observed decreased proliferation rate and changes in size/morphology of HSV-TKexpressing cells. Nevertheless, it has to be noted, that without 
GCV supplementation, Rh30TK cell line was continuously growing in contrast to Rh30TK cells in the presence of prodrug as shown on Fig. 3.

Obtained data showed efficient gap junction intercellular communication (GJIC) in Rh30 cell line. The co-culture of Rh30 unmodified cells with HSV-TK ${ }^{+}$modified cells using different cell ratios were performed to evaluate the efficiency of toxic bystander effect. The lowest ratio of HSV-TK-expressing cells to wild-type cells which was 1:4 (20\% of Rh30TK cells in co-culture) was sufficient to eliminate all cells in culture after ganciclovir addition, however, co-culture containing only $10 \%$ of HSV-TK ${ }^{+}$cells probably could lead to the same effect if ganciclovir administration time was longer than 8 days. Pictures of $10 \% \mathrm{Rh} 30 \mathrm{TK}$ co-culture after 8 days showed that only few cells survived and severe cytotoxic effect was visible. Nevertheless, similar results of bystander effect in different cancer cell lines were claimed by researchers in numerous publications $(9,15-18)$.

Bystander effect is desired as a method of unmodified cell eradication. On the other hand, it may be responsible for killing adjacent non-malignant cells, as toxic product of GCV conversion is diffused to cells surrounding modified cells. Thus, removal of tumor in suicide gene therapy procedure is associated with harm to non-malignant cells existing in tumor niche. However, it has to be noted, that similar side-effects occur also in conventional therapies in cancer treatment. Surgical intervention affects tissues surrounding cancer cells, which are partially resected with tumor. Analogous imprecision of action can be observed in radiation therapy where severe side-effects can occur. Moreover, another conventional treatment, chemotherapy, is connected with systemic toxicity and substantial distress for patient. Hence, it has to be considered if suicide gene therapy can be a method of reducing collateral damage, especially as a part of combined treatment to shorten the time of healing. Our in vivo experiments revealed small remaining connective tissues and no other tissues in sites of injection with $\mathrm{HSV}_{-} \mathrm{TK}^{+}$rhabdomyosarcoma cells were affected, however, this model is far from clinical condition.

It has to be mentioned, that there are two mechanisms of bystander effects. One is based on gap junction intercellular communication (GJIC), the other one is gap junction-independent. Gap junction-independent bystander effect may include transfer of apoptotic vesicles, exocytosis of cytotoxic factors from the GCV-treated HSV-TK ${ }^{+}$cells and enhanced cellular immune response in vivo, although it is likely that a synergism of these mechanisms occurs in a living organism (10). Conducted experiments proved both strong gap-junctional intercellular communication (GJIC) and efficient, toxic bystander effect in examined Rh30 cell line, which confirm literature data concerning this rhabdomyosarcoma cell line (19). Such results are very promising from the perspective of clinical application of suicide therapy as in vivo not only genetic modification efficacy is limiting, but also distribution of ganciclovir might be restricted.

The efforts to use suicide gene therapy in clinical application have met incomplete success. Preclinical and clinical studies using adeno- and retroviral vectors revealed relatively poor responses due to insufficient gene transfer and inefficient gene expression within tumors. The aim of in vivo approach in suicide gene therapy is to introduce suicide gene accurately to cancer cells. This can be achieved by in situ transfection or transduction, however, the efficiency of these methods remains relatively low in comparison to in vitro gene introduction. The ideal situation for suicide gene therapy would be to deliver HSV-TK gene as a vector targeting tumor systemically for the treatment of metastatic diseases. The promising concept is to use a virus genetically modified to carry a ligand for receptor present on the cancer cell surface (such as high affinity laminin receptor) (20). Nevertheless, introduction of suicide gene to cancer cells exclusively is still a demanding issue and a main obstacle in bringing suicide gene therapy to the clinic.

Efficient distribution of toxic product after ganciclovir administration was achieved, additionally, thanks to strong GJIC, we were able to eradicate Rh30 cells in co-culture in vitro. Interestingly, literature describes very low intercellular communication in the RD, another rhabdomyosarcoma cell line (21). Even in cells characterized by inefficient GJIC suicide gene therapy approach can be applied when combined with enhancement of GJIC by introduction of connexin transgene (22). The type of $\mathrm{Cx}$ expressed (by transfection or otherwise) does not appear to be crucial for the bystander effect because similar results were obtained with HeLa cells expressing various $\mathrm{Cx}$ (15). The most widely introduced Cx's, due to theirs versatility, are $\mathrm{Cx} 26$ or $\mathrm{Cx} 43$, as they assure efficient communication between cells obligatory for gap-junction dependent bystander effect to exist (23-25). However, another genetic modification complicates the procedure and, what is more important, is generally connected with higher toxicity level and cellular stress. Contrary to RD cell line, there was no need to upregulate connexins in examined Rh30 cell line. Different nature of these two cell lines, embryonal for RD and alveolar for Rh30 may be the possible explanation for the phenomenon of different connexin levels. Considering this, the perspective of usefulness of suicide gene therapy may depend on diagnosed rhabdomyosarcoma type.

Suicide gene therapy is also promising concept for eradication of cells resistant to conventional anticancer treatment. Cancer stem cells are believed to be resistant to chemo- and radio-therapy, and thus, responsible for recurrence of cancer after treatment (26). In suicide gene therapy approach this obstacle is overcome by efficient toxic bystander effect.

In our experiments only 14 days of GCV systemic administration was satisfactory for rhabdomyosarcoma xenografted tumors to be eliminated. The analysis of eradicated Rh30TK tumors discovered no existence of rhabdomyosarcoma cell aggregates, examined residues contained solely connective tissue and lymph nodes. Samples of organs, such as brain, liver, spleen and kidney were collected to assess possible in vivo toxicity of ganciclovir. The histopathological examination revealed no evidence of systemic toxic influence of ganciclovir. GCV action and side-effects are well known due to its widespread usefulness in antiviral therapy. Treatment with GCV is associated with a range of serious hematological adverse effects (27). In the present study, no toxic effects on various organ systems can be explained by low dose of GCV, short time required to eliminate growing tumors and short time of whole experiment in consequence. It is possible that multiorgan toxicity can affect animals if administration of GCV for longer period of time had been performed. On the other hand, adverse effects unable to be verified in a mouse model, but affecting human (fever, sweating, nausea, abdominal pain, 
headache, confusion, hallucination or seizures) have to be of concern during potential suicide gene therapy (27).

Our experiments were not planned to test in vivo elimination of Rh30 cell population containing low proportion of HSV-TK-positive cells. These data were only obtained in vitro and confirmed high efficiency of HSV-TK suicide gene strategy to eliminate all cancer cells even if modified cells were limited to $10 \%$ of the cell mixture. Mouse model was designed to evaluate the ability of this approach to rapid tumor growth inhibition and eradication of rhabdomyosarcoma cells by systemic (intraperitoneal) administration of ganciclovir. Despite this, collected data suggest that elimination of HSV-TK modified and wild-type cell mixture should be effective in a living organism as well. There are numerous data related to suicide gene therapy, indicating no considerable alterations in gap junction intercellular communication between in vitro and in vivo approaches (28). As we confirmed stable and efficient GJIC in investigated Rh30 rhabdomyosarcoma cell line in vitro, similar effects in a mouse model are expected.

In conclusion, we showed that suicide gene therapy involving HSV-TK + GCV in rhabdomyosarcoma can be very effective. Almost all tumor cells are eradicated in vitro when HSV-TK-expressing cells comprise $20 \%$ of the population. These results prove high efficiency of the therapy. Importantly, our approach is also effective in vivo, where we observed almost complete remission of tumors upon only 14 days of systemic administration of GCV. These results are very promising for future clinical application of suicide therapy. Although more research concerning effect of prolonged exposure to GCV and efficiency of tumor elimination upon low percentage of HSV-TK-expressing cells present within the tumor are necessary to establish security and efficiency of such treatment, suicide gene therapy is very promising concept in future clinical studies concerning rhabdomyosarcoma.

\section{Acknowledgements}

Authors would like to acknowledge the contribution of Dr Grażyna Drabik and Elżbieta Trześniowska-Popiel in histopathological analysis as well as Dr Kazimierz Węglarczyk and Dr Rafał Szatanek in cell sorting. The present study was supported by the National Science Centre grant no. N407 048538 and KNOW - Leading National Research Centre 2012-2017.

\section{References}

1. O'Brien D, Jacob AG, Qualman SJ and Chandler DS: Advances in pediatric rhabdomyosarcoma characterization and disease model development. Histol Histopathol 27: 13-22, 2012.

2. Ognjanovic S, Linabery AM, Charbonneau B and Ross JA: Trends in childhood rhabdomyosarcoma incidence and survival in the United States, 1975-2005. Cancer 115: 4218-4226, 2009.

3. Geltzeiler M, Li G, Abraham J and Keller C: The case for primary salivary rhabdomyosarcoma. Front Oncol 5: 74, 2015.

4. Kashi VP, Hatley ME and Galindo RL: Probing for a deeper understanding of rhabdomyosarcoma: Insights from complementary model systems. Nat Rev Cancer 15: 426-439, 2015.

5. Parham DM and Ellison DA: Rhabdomyosarcomas in adults and children: an update. Arch Pathol Lab Med 130: 1454-1465, 2006.

6.Malempati S and Hawkins DS: Rhabdomyosarcoma: Review of the Children's Oncology Group (COG) Soft-Tissue Sarcoma Committee experience and rationale for current COG studies. Pediatr Blood Cancer 59: 5-10, 2012.
7. Kashima K, Watanabe M, Sato Y, Hata J, Ishii N and Aoki Y: Inhibition of metastasis of rhabdomyosarcoma by a novel neutralizing antibody to $\mathrm{CXC}$ chemokine receptor-4. Cancer Sci 105: 1343-1350, 2014

8. Kokoris MS and Black ME: Characterization of herpes simplex virus type 1 thymidine kinase mutants engineered for improved ganciclovir or acyclovir activity. Protein Sci 11: 2267-2272, 2002.

9. Nicholas TW, Read SB, Burrows FJ and Kruse CA: Suicide gene therapy with Herpes simplex virus thymidine kinase and ganciclovir is enhanced with connexins to improve gap junctions and bystander effects. Histol Histopathol 18: 495-507, 2003.

10. Andrade-Rozental AF, Rozental R, Hopperstad MG, Wu JK, Vrionis FD and Spray DC: Gap junctions: The 'kiss of death' and the 'kiss of life'. Brain Res Brain Res Rev 32: 308-315, 2000.

11. Peiris TH and Oviedo NJ: Gap junction proteins: Master regulators of the planarian stem cell response to tissue maintenance and injury. Biochim Biophys Acta 1828: 109-117, 2013.

12. Nielsen MS, Axelsen LN, Sorgen PL, Verma V, Delmar M and Holstein-Rathlou NH: Gap junctions. Compr Physiol 2: 1981-2035, 2012.

13. Czyż J, Irmer U, Schulz G, Mindermann A and Hülser DF: Gap-junctional coupling measured by flow cytometry. Exp Cell Res 255: 40-46, 2000

14. Di Ianni M, Di Florio S, Venditti G, Falzetti F, Mannoni P, Martelli MF and Tabilio A: T lymphocyte transduction with herpes simplex virus-thymidine kinase (HSV-tk) gene: Comparison of four different infection protocols. J Hematother Stem Cell Res 8: 645-652, 1999.

15. Mesnil $\mathrm{M}$ and Yamasaki H: Bystander effect in herpes simplex virus-thymidine kinase/ganciclovir cancer gene therapy: Role of gap-junctional intercellular communication. Cancer Res 60: 3989-3999, 2000

16. You Y: Targets in Gene Therapy. InTech, 2011. http://dx.doi. org/10.5772/1012.

17. Neschadim A, Wang JC, Lavie A and Medin JA: Bystander killing of malignant cells via the delivery of engineered thymidine-active deoxycytidine kinase for suicide gene therapy of cancer. Cancer Gene Ther 19: 320-327, 2012.

18. Veldwijk MR, Berlinghoff S, Laufs S, Hengge UR, Zeller WJ, Wenz F and Fruehauf S: Suicide gene therapy of sarcoma cell lines using recombinant adeno-associated virus 2 vectors. Cancer Gene Ther 11: 577-584, 2004.

19. Wildner O, Morris JC, Vahanian NN, Ford H Jr, Ramsey WJ and Blaese RM: Adenoviral vectors capable of replication improve the efficacy of HSVtk/GCV suicide gene therapy of cancer. Gene Ther 6: 57-62, 1999.

20. Tseng JC, Zanzonico PB, Levin B, Finn R, Larson SM and Meruelo D: Tumor-specific in vivo transfection with HSV-1 thymidine kinase gene using a Sindbis viral vector as a basis for prodrug ganciclovir activation and PET. J Nucl Med 47: 1136-1143, 2006.

21. Lin Z, Zhang Z, Han Y, Naus CC, Yu KR and Holtzer H: Functional expression of gap junction gene $\mathrm{Cx} 43$ and the myogenic differentiation of rhabdomyosarcoma cells. Sci China B 38: 305-312, 1995.

22. Mesnil M: Connexins and cancer. Biol Cell 94: 493-500, 2002.

23. Garcia-Rodríguez L, Pérez-Torras S, Carrió M, Cascante A, García-Ribas I, Mazo A and Fillat C: Connexin-26 is a key factor mediating gemcitabine bystander effect. Mol Cancer Ther 10: 505-517, 2011.

24. Sanson M, Marcaud V, Robin E, Valéry C, Sturtz F and Zalc B: Connexin 43-mediated bystander effect in two rat glioma cell models. Cancer Gene Ther 9: 149-155, 2002.

25. Tanaka M, Fraizer GC, De La Cerda J, Cristiano RJ, Liebert M and Grossman HB: Connexin 26 enhances the bystander effect in HSVtk/GCV gene therapy for human bladder cancer by adenovirus/PLL/DNA gene delivery. Gene Ther 8: 139-148, 2001.

26. Ciurea ME, Georgescu AM, Purcaru SO, Artene SA, Emami GH, Boldeanu MV, Tache DE and Dricu A: Cancer stem cells: Biological functions and therapeutically targeting. Int J Mol Sci 15: 8169-8185, 2014.

27. CYTOVENE ${ }^{\circledR}$ Product Monograph. http://www.rochecanada. $\mathrm{com} / \mathrm{en} /$ products/pharmaceuticals/consumer information/ cytovene.html.

28. van Dillen IJ, Mulder NH, Vaalburg W, de Vries EFJ and Hospers GAP: Influence of the bystander effect on HSV-tk/GCV gene therapy. A review. Curr Gene Ther 2: 307-322, 2002. 